\title{
Über eine Makrostruktur des amorphen Selens
}

\author{
Von Joachim Weber \\ Aus dem Standard Laboratorium der Süddeutschen Apparate-Fabrik, Nürnberg \\ (Z. Naturforschg. 8a, 565-566 [1953]; eingegangen am 16. Juli 1953) \\ Bekanntlich kann amorphes Selen eine Makrostruktur zeigen, die einen kristallinen \\ Aufbau vortäuscht. Die Bedingungen, unter denen diese Struktur auftreten kann, werden \\ angegeben. Abbildungen bei verschiedenen Vergrößerungen sollen einen Einblick in die \\ Vorgänge bei der Entstehung einer amorphen Selenschicht mit bevorzugter Orientierung
} geben.

$I^{n}$ einer Arbeit über das amorphe Arsen, welche sich in erster Linie mit der Feinstruktur des amorphen Arsens befaßt, berichtet Richter ${ }^{1}$ über eine Stäbchenstruktur an aufgedampften amorphen Arsenschichten.

Wir fanden bei der Untersuchung aufgedampfter Selenschichten gelegentlich ähnliche Erscheinungsformen und stellten dem genannten Verfasser für eine entsprechende Arbeit über das amorphe Selen ${ }^{2}$ die photographische Aufnahme einer amorphen Selenprobe mit ausgesprochener Stäbchenstruktur zur Verfügung. Es erscheint uns nützlich, einerseits über die Bedingungen zu berichten, unter denen derartige Strukturen beim Aufdampfen von Selen entstehen können, und andererseits an einigen Mikroaufnahmen den Aufbau der Selenschicht zu zeigen.

\section{Bedingungen für die Entstehung der Stäbchenstruktur}

Das Auftreten einer Stäbchenstruktur wurde von uns mehrmals an der in Abb. 1 wiedergegebenen Bedampfungsanordnung beobachtet. Die „Stäbchenpakete" bildeten sich an einer wassergekühlten blankgezogenen Messingfläche, welche vor der Bedampfung mit Öl in Berührung gekommen war. Sie erhielt durch den rhythmisch sich darunter bewegenden Verdampfer eine Reihe von Bedampfungsimpulsen. Die Zeitdauer für einen Impuls war etwa $3 \mathrm{~s}$, die Bedampfungslücke $50 \mathrm{~s}$, wobei bei einem Totaldruck von $10^{-4}$ Torr im Mittel je Impuls 1,7 mg Selen pro $\mathrm{cm}^{2}$ kondensiert wurden.

Das im Verdampfer befindliche Selen enthielt als Fremdbestandteil $5 \cdot 10^{-5}$ Gewichtsanteile Jod. Den gleichen Jodgehalt wies auch das Selen der Stäb-

1 H. Richter u. G. Breitling, Z. Naturforschg. 6 a, 721 [1951]; Abb. 2 s. Tafel S. 724 b.

${ }^{2}$ H. Richter, W. Kulcke u. H. Specht, Z. Na- chenpakete auf. Nach durchschnittlich zwölf Impulsen wurde eine längere Pause eingelegt, wobei die Apparatur geöffnet wurde.

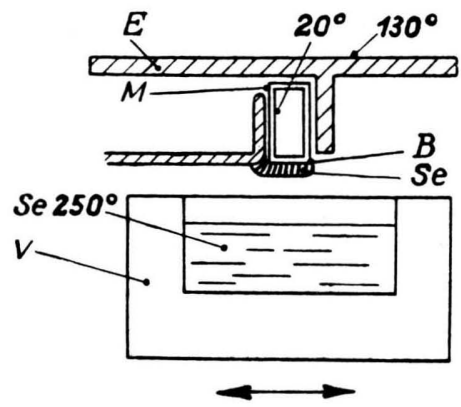

Abb. 1. Versuchsanordnung. E Eisenblech, B Basisfläche, M Messingrohr $16 \times 8$, Se Selen, $\mathrm{Se}_{250}{ }^{0}$ Selen $250^{\circ}$, V Verdampfer, $\longleftrightarrow$ Bewegungsrichtung des Verdampfers.

\section{Untersuchung der Stäbchenstruktur}

In Abb. 2* wird ein Stäbchenpaket gezeigt, welches von der Basis abgelöst wurde. Vorn ist eine Bruchfront zu sehen, an der die einzelnen Stäbchen mit kristallähnlicher Struktur zu erkennen sind. Die Unterseite war dem Verdampfer zugewendet. Diese Wachstumsfront besteht aus einer Vielzahl von Kuppen, in die die senkrecht zur Basis parallel orientierten Stäbchen von 3,8 mm Länge auslaufen.

Die Proben sind parallel zur Wachstumsrichtung gut spaltbar.

In Abb. 3 wird eine besonders glatte Bruchfront an einem Stäbchenpaket bei einer mikroskopischen Vergrößerung von 1:8 gezeigt.

Man kann deutlich eine durchgehende Schichtung senkrecht zur Wachstumsrichtung erkennen. An der

turforschg. 7 a, 511 [1952]; Abb. 14a s. Tafel S. 516 a, Anm. 33a.

* Abb. 2-5, s. Tafel S. 566 a. 
linken Seite der glatten Bruchfront ist die Krümmung der Basisfläche und die Orientierung der Stäbchen in Richtung des Krümmungsradius sichtbar. Unmittelbar an der Basis erscheint die Struktur verwischt und feinkörnig.

In Abb. 4 wird eine glatte Bruchfront bei einer mikroskopischen Vergrößerung von 1:150 gezeigt. In dieser Abbildung ist die Aufteilung der einzelnen Stäbchen in eine Vielzahl von Fasern sehr deutlich zu erkennen.

Außerdem ist die Aufeinanderfolge der einzelnen Impulse und der verschiedenen Bedampfungen mit großer Genauigkeit abzulesen. Diese Aufnahme läßt bereits Schlüsse auf das Entstehen der Stäbchen zu. Man sieht, daß jede Faser aus einer Reihe paralleler ,Tröpfchenketten“ besteht. Die Ketten hängen im allgemeinen lotrecht zur Basisfläche. Mitunter treten schwache Abweichungen von dieser Richtung auf, die aber bei der nächsten Bedampfung wieder ausgeglichen werden. Charakteristisch für den Wachstumsprozeß ist die Tatsache, daß über viele Bedampfungen hinweg die Tröpfchen sich in der Weise aneinanderhängen, daß verhältnismäßig lange parallele Ketten entstehen. Diese Ketten liegen eng aneinander und bilden ein Bündel, in welchem sich die Tröpfchen, die aus dem gleichen Impuls stammen, auch seitlich berühren. In der Schicht, die den Anschluß an die vorhergehende Bedampfung bildet, sind die Tröpfchen verzerrt, während sie in der darauffolgenden Schicht bereits dieselbe Form haben wie in der Mitte der Impulsreihe. Eine lineare Vergrößerung der Aufnahme in Abb. 4 auf 1:6, entsprechend einer Gesamtvergrößerung von 1:900, zeigt diese Ketten sehr deutlich (Abb. 5).
Von mehreren Stäbchen mit etwa 0,6 mm Durchmesser wurden Debye-Scherrer-Aufnahmen mit Cu$\mathrm{K} \alpha$-Strahlung gemacht, wobei die Stäbchen um ihre Längsachse gedreht wurden mit dem Ergebnis, daß keine Interferenzen auftraten. Obwohl die Proben ein kristallähnliches Aussehen haben, sind sie amorph. Die gleichen Stäbchen ergaben nach einer Temperzeit von $30 \mathrm{~min}$ bei $217^{\circ} \mathrm{C}$ scharfe Interferenzen. Die Probe wurde dabei feinkristallin und grau; die ursprüngliche Makrostruktur verwischte. Für die Entstehung dieser auffälligen Makrostruktur kann eine befriedigende Erklärung vorläufig nicht gegeben werden. Man könnte annẹmen, daß, durch eine dünne Adsorptionsschicht auf der Grundplatte begünstigt, bei den gegebenen Temperaturbedingungen das Selen die Basis nicht gleichmäßig benetzt hat, so daß sich auf der Kondensationsfläche ein Raster aus Selentröpfchen bilden konnte. An diese Tröpfchen haben sich dann bei jedem folgenden Impuls Schicht für Schicht weiterhin Tröpfchen angehängt in der Weise, daß die in den gezeigten Aufnahmen erkennbaren Ketten entstanden sind. Es ist möglich, daß auch der Jodgehalt des Selens in dem von Richter in der bereits erwähnten Arbeit angedeuteten Sinne die Ausbildung der Stäbchenstruktur begünstigt ${ }^{3}$. Rich ter nimmt an, daß bei Gegenwart von Fremdstoffen diese nicht allein die freien Valenzen abgebrochener Ketten absättigen, sondern zudem die Se-Ketten schlauchartig umhüllen und die Parallelorientierung begünstigen. In dieser Parallelorientierung im Feinbau wäre dann letzten Endes die Ursache für die Ausbildung der gezeigten Makrostruktur zu sehen.

\footnotetext{
${ }^{3}$ 1. c. ${ }^{2}$ S. 530.
} 


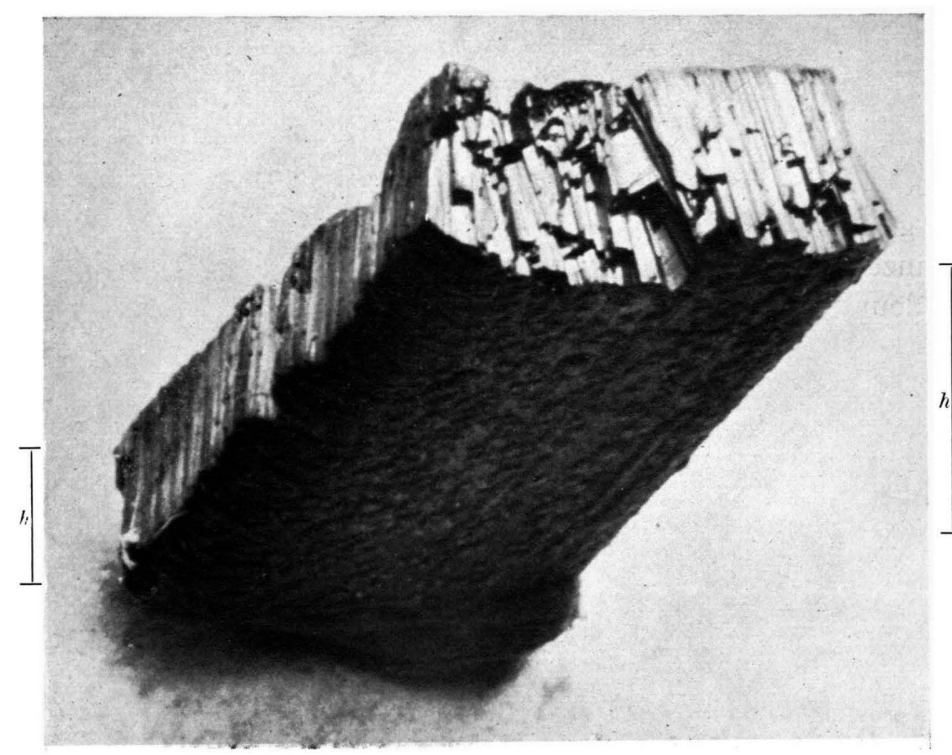

Abb. 2. Von der Basis abgelöstes Stäbchenpaket. $h=3,8 \mathrm{~mm}$.

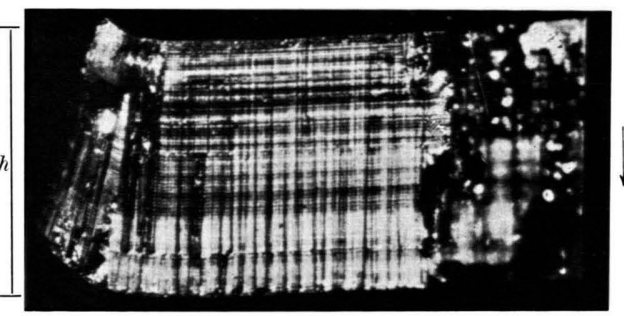

Abb. 3. Bruchfront an einem Stäbchenpaket. Mikroskopische Vergr. 1:8. $h=$ $3,8 \mathrm{~mm}$. Pfeil gibt Wachstumsrichtung an.

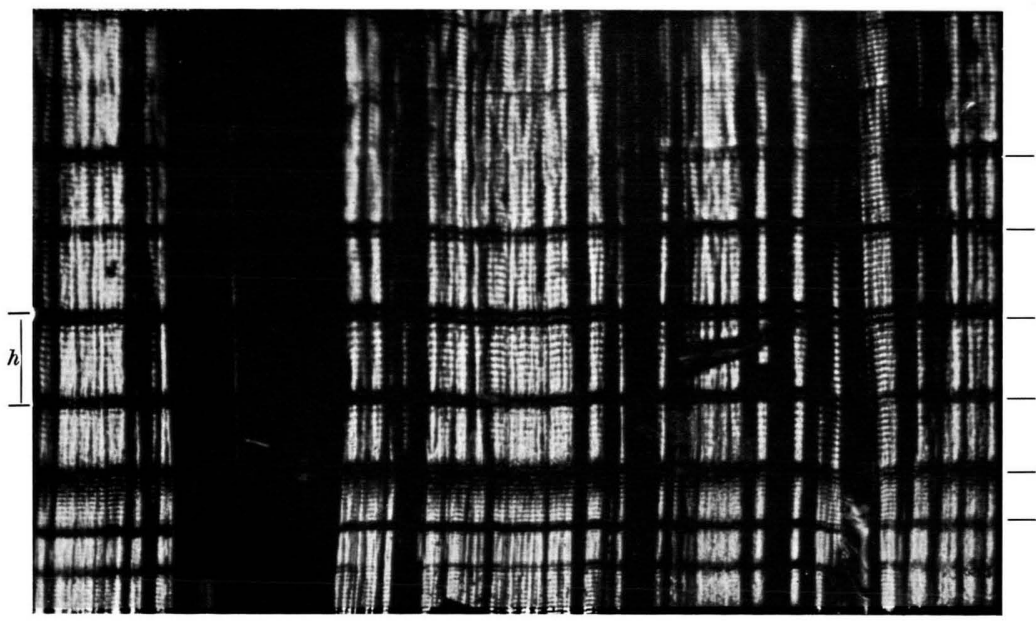

Abb. 4. Bruchfront. Mikroskopische Vergr. $1: 150 . h=40 \mu$. Striche weisen auf die Pausen zwischen den Bedampfungen hin.

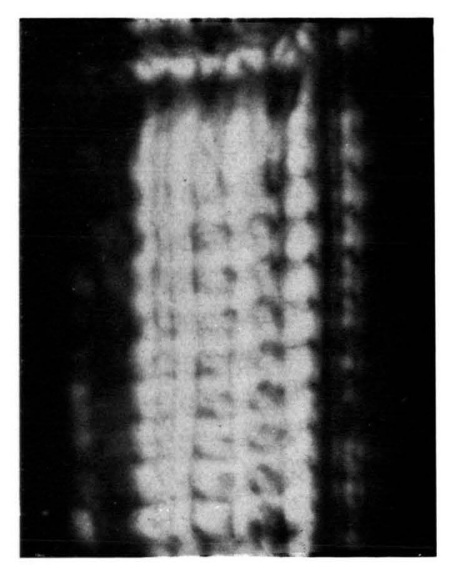

Abb. 5. Lineare Vergr. der Aufnahme in Abb. 4 auf 1:6. Gesamtvergr. $1: 900$. 


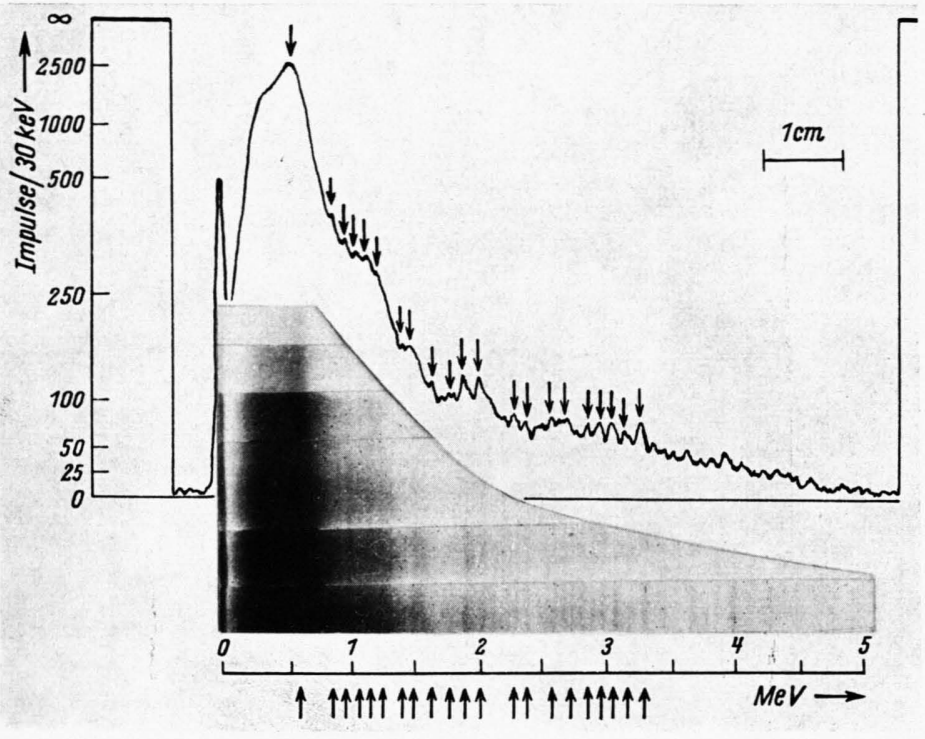

Abb. 3. Aufnahme und Photometerkurve der Energieverteilung der Stickstoff-Reaktionsprodukte (84000 Impulse).

Abb. 4. Aufnahmen und Photometerkurven der Energieverteilung der Sauerstoff-Reaktionsprodukte(obereAufnahme 38000 Impulse, untere Aufnahme 256000 Impulse).

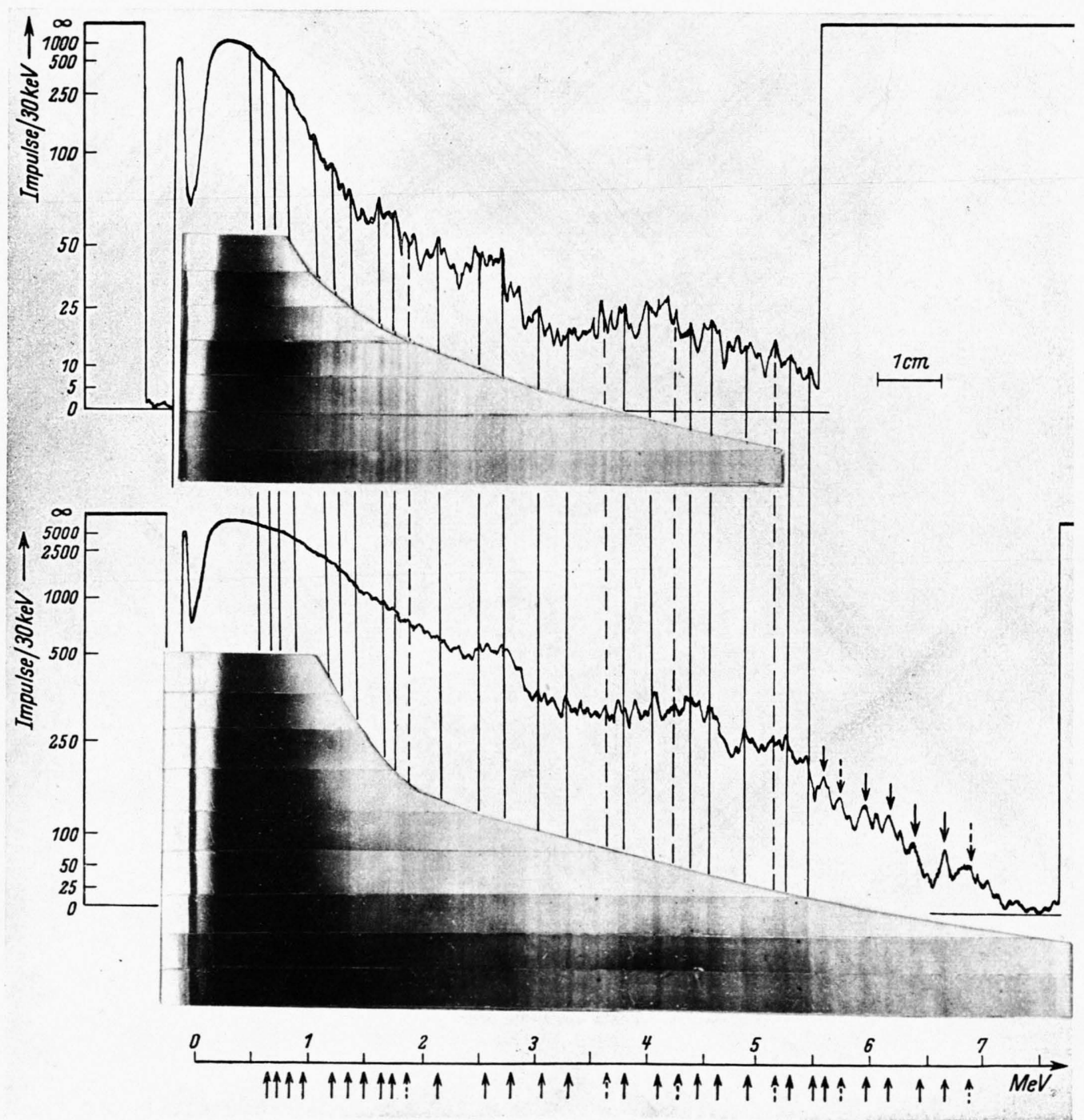

